Article

\title{
Development of a Magnetostrictive FeNi Coated Surface Acoustic Wave Current Sensor
}

\author{
Jie Tong ${ }^{1}$, Yana Jia ${ }^{2,3}$, Wen Wang ${ }^{2, *}$, Yang Wang ${ }^{1}$, Shiyue Wang ${ }^{1}$, Xinlu Liu ${ }^{2}$ and Yuqing Lei ${ }^{1}$ \\ 1 China Electric Power Research Institute of State Grid, Beijing 100192, China; tongjie1@epri.sgcc.com.cn (J.T.); \\ yangw@epri.sgcc.com.cn (Y.W.); 15121479@bjtu.edu.cn (S.W.); yuqinglei@epri.sgcc.com.cn (Y.L.) \\ 2 State Key Laboratory of Acoustics, Institute of Acoustics, Chinese Academy of Sciences, No. 21, \\ Beisihuan West Road, Beijing 100190, China; jiayana225@163.com (Y.J.); liuxinlu1987@foxmail.com (X.L.) \\ 3 School of Electronic, Electrical and Communication Engineering, University of Chinese Academy of \\ Sciences, Beijing 100049, China \\ * Correspondence: wangwenwq@mail.ioa.ac.cn; Tel.: +86-10-8254-7803
}

Received: 7 July 2017; Accepted: 20 July 2017; Published: 25 July 2017

Featured Application: The developed magnetostrictive FeNi coated Surface acoustic wave sensor with high sensitivity; fast response; strong anti-interference; good linearity and repeatability; and lower hysteresis error will be a potential candidate for current monitoring application in smart grid line testing; metallurgical and power supplies; rail transit safety warnings and rescue.

\begin{abstract}
A magnetostrictive FeNi-coated surface acoustic wave (SAW)-based current sensor was proposed in this work. The weak remanence and hysteresis effect of the FeNi itself contributes to suppress the asymmetry in sensor response at increasing and decreasing current. The sensor response was simulated by solving the coupled electromechanical field equation in layered structure considering the magnetostrictive effect and an approach of effective dielectric constant. The effects from the aspect ratio and thickness of the FeNi film on sensor response were analyzed to determine the optimal design parameters. Differential oscillation structure was used to form the sensor, in which, the FeNi thin film was deposited along the SAW propagation of the sensor chip by using RF magnetron sputtering. The magnetostrictive effect of the FeNi coating induced by the magnetic loading generates the perturbation in SAW velocity, and corresponding oscillation frequency. High sensitivity of $10.7 \mathrm{KHz} / \mathrm{A}$, good linearity and repeatability, lower hysteresis error of $0.97 \%$ were obtained from the developed prototype $150 \mathrm{MHz}$ SAW FeNi coated current sensor.
\end{abstract}

Keywords: current sensor; differential oscillator; FeNi; magnetomechanics effect; surface acoustic wave

\section{Introduction}

Surface acoustic wave (SAW)-based devices attracts increasing attention for various physical and chemical sensing because they offer some unique performances such as high sensitivity, excellent reproducibility, small size, simple structure, robustness, being passive (batteryless), and remotely queried (wireless). Many proven SAW-based sensors were developed for sensing gas species, temperature, force, torque, and so on [1-5]. Recently, referring to the magnetostrictive thin film as the sensing material or magnetoresistor as the external loaded sensor, a new concept of SAW-based magnetic/current sensor were proposed, allowing possible features of fast response, high sensitivity, strong anti-interference, and small size. The functional magnetostrictive thin-film materials are favored and very promising for high-precision magnetic/current field measurement owing to their large magnetostrictive coefficient, high energy conversion efficiency, fast response and non-contact driving. Kodota et al. subsequently presented a new prototype of SAW magnetic sensor using interdigital 
transducers (IDTs) made by magnetostrictive Ni electrodes on a quartz substrate and wireless and passive measurement was offered. Obtained magnetic sensitivity was $730 \mathrm{~Hz} / \mathrm{mT}[6,7]$. Elhosni et al. proposed a similar SAW magnetic sensor with a magnetostrictive-piezoelectric layered structure of $\mathrm{Ni} / \mathrm{ZnO} / \mathrm{IDT} / \mathrm{LiNbO}_{3}$, and the corresponding finite element modeling (FEM) simulation was performed to predict the sensor performance [8,9]. Using the Galfenol thin film as the sensitive interface, a SAW magnetic field sensor was developed by Li et al. a maximum change of $0.64 \%$ in SAW velocity was obtained with a $500 \mathrm{~nm}$ Galfenol thick film, but not including further details about the experimental results [10]. Zhou et al. addresses the experimental and theoretical investigations of SAW magnetic sensor structured by $\mathrm{TbCo}_{2} / \mathrm{FeCo} / \mathrm{Y}$-cut $\mathrm{LiNbO}_{3}$ [11]. Just recently, a magnetostrictive FeCo-coated SAW magnetic/current sensor using the differential oscillation structure was proposed in our previous work, where high sensitivity of $16.6 \mathrm{KHz} / \mathrm{A}$ was obtained [12].

As for the magnetoresistor loaded magnetic sensor, Hauser et al. developed a wirelessly interrogable micro-magnetic field sensor using the giant magneto-impedance (GMI) effect in a $30 \mu \mathrm{m}$ diameter amorphous FeCoSiBNd wire of zero magnetostriction, and a SAW transponder devices, a relative signal sensitivity of $80 \mathrm{~dB} / \mathrm{T}$ at low magnetic flux density B less than $30 \mathrm{mT}$, quick response, and a high temperature stability were obtained [13]. Utilizing a reflective delay line pattern loaded a tri-layer GMI sensor, an autonomous magnetic field sensor was realized by Li et al. and a sensitivity of $0.34 \mathrm{~dB} /$ Oe are reported experimentally $[14,15]$. Also, Reindl et al. reported a dexterous design of the configuration of the SAW magnetic/current sensor loaded with magnetoresistor sensor, where wireless and passive measurement for current was realized [16,17], and the obtained current resolution is approximately $5 \%$ of full scale $(-800 \sim 800 \mathrm{~A})$.

Obviously, the magnetostrictive film coated SAW sensor exhibits simpler design, higher magnetic sensitivity and better temperature compensation in sensor structure over the magnetoresistor loaded sensor, and becomes the future research focus. Some magnetostrictive materials with larger magnetostrictive coefficient as $\mathrm{FeCo}, \mathrm{TbCo}_{2}$ were advised as the sensitive coating, which produce higher magnetic sensitivity. However, due to the large magnetic remanence and hysteresis effect of these materials mentioned above, there occurs obvious asymmetry in sensor response at increasing and decreasing current, which degrades significantly the sensor performance.

Among the available magnetostrictive materials, the FeNi features very small variation of coercive field and narrow and steep magnetic hysteresis, and additionally, it has excellent rust resistance and better process ability over the FeCo film, which makes it very promising for magnetic/current sensing, and was employed as the sensitive materials in this contribution.

So, the first aim in this work is to perform the simulation on the sensor response by solving the coupled electromechanical field equation in layered structure considering the magnetostrictive effect and approach of effective constant [12], and then to determine the optimal design parameters for FeNi coating preparation by analyzing the effect on sensor response from the film thickness and aspect ratio.

Then, a FeNi-coated SAW based current sensor was constructed, which was composed of a differential SAW delay line oscillator configuration, and magnetostrictive FeNi thin film deposited along the SAW propagation path of the sensing device. The scheme and working principle of the proposed sensor are depicted in Figure 1. $\mathrm{YZ}-\mathrm{LiNbO}_{3}$ was utilized as the piezoelectric substrate, which provides larger electromechanical coupling coefficient $(4.1 \%)$ to reduce the insertion loss of the SAW chip, and linear temperature coefficient of frequency (TCF) contributing to the temperature compensation by using the differential structure mentioned in Figure 1. Additionally, the single phase unidirectional transducers (SPUDTs) with Al electrodes were used to structure the SAW device to reduce the insertion loss $[18,19]$. The FeNi thin film was then coated on the top surface of the sensing device by RF magnetron sputtering and overlay technique. Prior to FeNi coating, a $50 \mathrm{~nm} \mathrm{SiO} 2$ film was deposited on the piezoelectric substrate to provide a well protection on interdigital transducers (IDTs) in procedure of FeNi coating. The FeNi film produces magnetostrictive strain stimulated by the electromagnetic field, where the thickness, density and young's modulus of the FeNi film are changed accordingly, and then the SAW propagation characteristic are modulated in the form of shift in SAW 
velocity. The applied electromagnetic field can be evaluated by using the corresponding differential oscillation frequency signal. Utilizing the Helmholtz coil system, the developed SAW sensor was characterized, and in comparison to FeCo coated sensor.

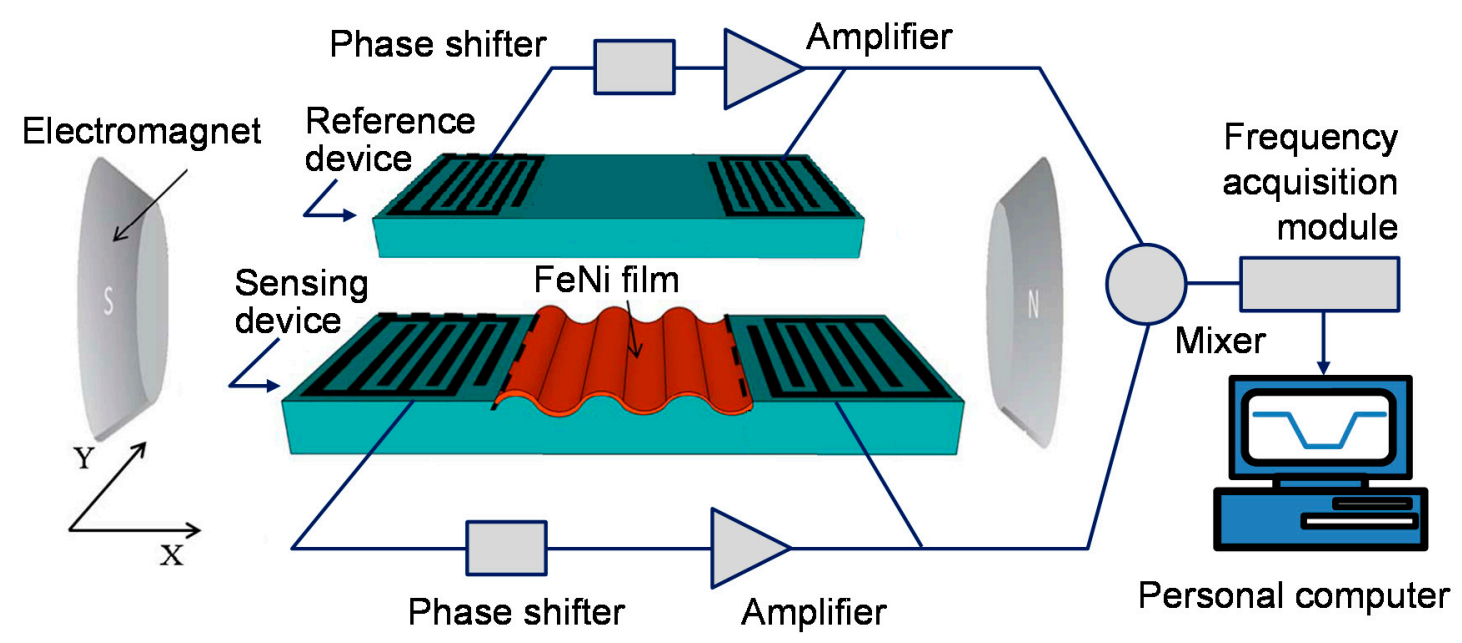

Figure 1. The scheme and working principle of the surface acoustic wave (SAW) current sensor.

\section{Simulation on the Sensor Response}

Prior to sensor device development, the sensor response was simulated theoretically to extract the optimal design parameters.

In the simulation, the SAW sensor was simplified as a structure composed of a semi-infinite piezoelectric substrate with IDT pattern, a $\mathrm{SiO}_{2}$ coating, and a magnetostrictive FeNi thin film as shown in Figure 2. Also, the theoretical simulation is referring to the way of an analytical resolution of acoustic motion equations in layered structure considering the magnetostrictive effect described in [12], so the influences of different parameters on sensor performances appear clearly. The corresponding coordinate system is shown in Figure 2. Acoustic wave propagates along the $x_{1}$ axis on the $x_{1}-$ $x_{2}$ plane at $x_{3}=0$. All material parameters of the mediums are transformed into the coordinate system. Each material in the layered structure is defined by its physical parameters: stiffness constants $\left(c_{i j k l}^{I}\right)$, piezoelectric constant $\left(e_{k i j}\right)$, permittivity constant $\left(\varepsilon_{i j}\right)$, and density $\left(\rho^{I}\right)$ for $\mathrm{LiNbO}_{3}$, stiffness constants $\left(\left(c^{I I} i j k l\right)\right)$ and density $\left(\rho^{I I}\right)$ for $\mathrm{SiO}_{2}$, Young's modulus $(E)$ and density $\left(\rho^{I I I}\right)$ for FeNi. $h_{1}$ and $h_{2}$ express the thicknesses of $\mathrm{SiO}_{2}$ and $\mathrm{FeNi}$, respectively. The corresponding physical parameters for piezoelectric substrate, $\mathrm{SiO}_{2}$, and $\mathrm{FeNi}$ are listed in Table 1 [20]. The SAW velocity relating to the electromagnetic field intensity can be calculated by solving the elastic wave equations of each media utilizing corresponding boundary conditions. The following is the specific theoretical simulation.

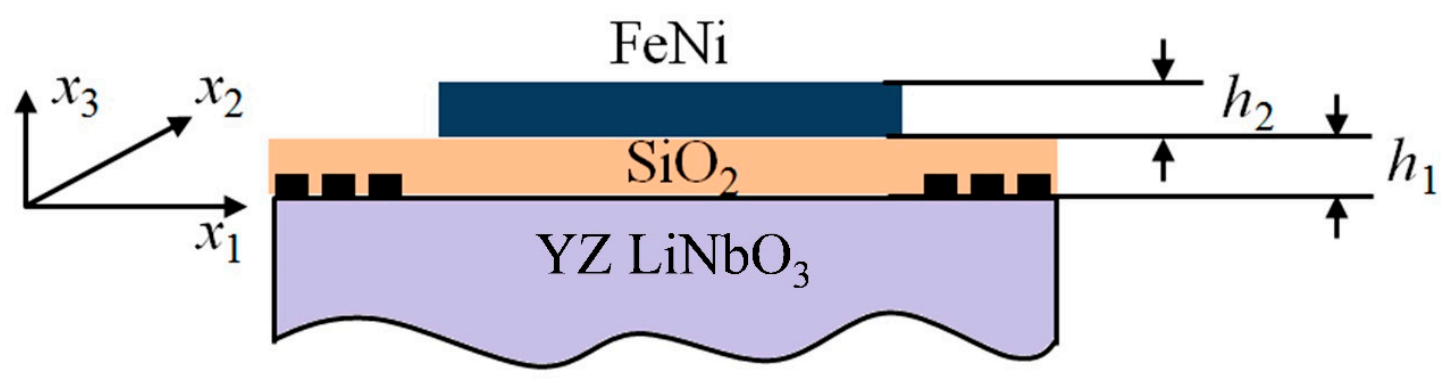

Figure 2. The corresponding coordinate system in this work. 
Table 1. Physical parameters of the piezoelectric substrate, $\mathrm{SiO}_{2}$, and FeNi.

\begin{tabular}{|c|c|c|c|c|}
\hline \multirow{2}{*}{ Materials Parameters } & & \multicolumn{3}{|c|}{ Values } \\
\hline & & $\mathrm{LiNbO}_{3}$ & $\mathrm{SiO}_{2}$ & FeNi \\
\hline \multirow{6}{*}{ Stiffness Coefficients $\left(10^{11} \mathrm{~N} \mathrm{~m}^{-2}\right)$} & $C_{11}$ & 2.03 & 0.785 & 1.077 \\
\hline & $C_{12}$ & 0.53 & & 0.46 \\
\hline & $C_{13}$ & 0.75 & & \\
\hline & $C_{14}$ & 0.09 & & \\
\hline & $C_{33}$ & 2.45 & & \\
\hline & $\mathrm{C}_{44}$ & 0.6 & 0.312 & 0.307 \\
\hline \multirow{4}{*}{ Piezoelectric Modules $\left(\mathrm{C} \mathrm{m}^{-2}\right)$} & $e_{31}$ & 0.2 & & \\
\hline & $e_{15}$ & 3.7 & & \\
\hline & $e_{33}$ & 1.3 & & \\
\hline & $e_{22}$ & 2.5 & & \\
\hline \multirow{2}{*}{ Permittivity Constants $\left(10^{-11} \mathrm{~F} \mathrm{~m}^{-1}\right)$} & $\varepsilon_{11}$ & 84 & & \\
\hline & $\varepsilon_{33}$ & 29 & & \\
\hline Density $\left(\mathrm{kg} / \mathrm{m}^{3}\right)$ & & 7450 & 2651 & 8.38 \\
\hline \multicolumn{2}{|c|}{ Magnetostrictive Constant $\left(10^{-6}\right)$} & & & 400 \\
\hline \multicolumn{2}{|l|}{ Young's Modulus $\left(10^{9} \mathrm{~N} / \mathrm{m}^{2}\right)$} & & & 80 \\
\hline
\end{tabular}

It is well known that the constitutive equations in a piezoelectric medium are expressed as in [21].

$$
\left\{\begin{array}{c}
\rho \frac{\partial^{2} u_{i}}{\partial t^{2}}-\nabla_{i K} c_{K L}^{I} \nabla_{L j} u_{j}-\nabla_{i K} e_{K j} \nabla_{j} \Phi=0 \\
\nabla_{i} e_{i L}\left(\nabla_{L j} u_{j}\right)-\nabla_{i} \varepsilon_{i j} \nabla_{j} \Phi=0
\end{array}\right.
$$

where $i, j=1,2,3, K, L=1,2, \ldots 6, \rho, u_{i}$ and $\Phi$ are the density, displacement and electric potential in $\mathrm{YZ}-\mathrm{LiNbO}_{3}$, respectively.

The field space of the frequency domain and the frequency slowness field (s) can be characterized by Fourier transform pairs, hence, a general solution of the coupled wave Equation (1) was assumed in the slowness (s) region as

$$
\left\{\begin{array}{c}
\widetilde{u}_{i}^{I}\left(s, x_{3}\right)=A_{i}^{I} \exp \left(-j \alpha^{I} \omega s x_{3}\right) \\
\widetilde{\Phi}^{I}\left(s, x_{3}\right)=A_{4}^{I} \exp \left(-j \alpha^{I} I \omega s x_{3}\right)
\end{array}\right.
$$

where, $s$ is the SAW slowness, and defined by the reciprocal of the SAW velocity, $\alpha$ is the acoustic attenuation factor in $x_{3}$ direction.

Substituting Equation (2) into the coupling wave Equation (1), and the Christoffel equation of the piezoelectric substrate in the slowness field is obtained, which is a quadratic polynomial of $\alpha$, and expressed by

$$
\begin{gathered}
\left((\alpha)^{2} \boldsymbol{R}_{1}^{I}+\alpha \boldsymbol{R}_{2}^{I}+\boldsymbol{R}_{3}^{I}\right)\left(\begin{array}{c}
A_{1} \\
A_{2} \\
A_{3} \\
A_{4}
\end{array}\right)=0 \\
\boldsymbol{R}_{1}^{I}=\left(\begin{array}{cccc}
c_{55} & c_{54} & c_{53} & e_{z 5} \\
c_{45} & c_{44} & c_{43} & e_{z 4} \\
c_{35} & c_{34} & c_{33} & e_{z 3} \\
e_{z 5} & e_{z 4} & e_{z 3} & -\varepsilon_{z z}
\end{array}\right) \boldsymbol{R}_{2}^{I}=\left(\begin{array}{cccc}
c_{15}+c_{51} & c_{14}+c_{56} & c_{13}+c_{55} & e_{z 1}+e_{x 5} \\
c_{65}+c_{41} & c_{64}+c_{46} & c_{63}+c_{45} & e_{z 6}+e_{x 4} \\
c_{55}+c_{31} & c_{54}+c_{36} & c_{53}+c_{35} & e_{z 5}+e_{x 3} \\
e_{z 1}+e_{x 5} & e_{z 6}+e_{x 4} & e_{z 5}+e_{x 3} & -\varepsilon_{z x}-\varepsilon_{x z}
\end{array}\right)
\end{gathered}
$$




$$
\boldsymbol{R}_{3}^{I}=\left(\begin{array}{cccc}
c_{11}-\frac{\rho}{s^{2}} & c_{16} & c_{15} & e_{x 1} \\
c_{61} & c_{66}-\frac{\rho}{s^{2}} & c_{65} & e_{x 6} \\
c_{51} & c_{56} & c_{55}-\frac{\rho}{s^{2}} & e_{x 5} \\
e_{x 1} & e_{x 6} & e_{x 5} & -\varepsilon_{x x}
\end{array}\right)
$$

where, $\alpha^{I}$ is the characteristic root, and $A_{1,2,3,4}^{I}$ are undetermined constants, i.e., eigenvectors.

The necessary condition for the existence of the non-trivial solution in Christoffel equation is $\operatorname{det}(\Gamma)=0$, and an eight degree equation about $\alpha^{I}$ was obtained. $\Gamma$ is the determinant of the coefficient matrix. To ensure the decrease of the acoustic displacement into the substrate, four roots of $\alpha^{I}$ with imaginary part more than zero were picked, that is, $\alpha_{n}^{I}(n=1,2,3,4)$. Then, Substituting $\alpha_{n}^{I}$ into the Christoffel equation, a set of eigenvectors corresponding to each $\alpha^{I}$ are deduced. It is clear that only the linear superposition of the solutions as Equation (2) corresponding to $\alpha_{n}^{I}(n=1,2,3,4)$ can satisfy the Christoffel equation and the boundary conditions. Thus, the integral solution was formed as the linear superposition of the wavelet solution, that is,

$$
\left(\begin{array}{l}
\widetilde{u}_{1}^{I}\left(s, x_{3}\right) \\
\widetilde{u}_{2}^{I}\left(s, x_{3}\right) \\
\widetilde{u}_{3}^{I}\left(s, x_{3}\right) \\
\widetilde{\Phi}^{I}\left(s, x_{3}\right)
\end{array}\right)=\left(\begin{array}{cccc}
A_{11}^{I} & A_{12}^{I} & A_{13}^{I} & A_{14}^{I} \\
A_{21}^{I} & A_{22}^{I} & A_{23}^{I} & A_{24}^{I} \\
A_{31}^{I} & A_{32}^{I} & A_{33}^{I} & A_{34}^{I} \\
A_{41}^{I} & A_{42}^{I} & A_{43}^{I} & A_{44}^{I}
\end{array}\right)\left(\begin{array}{c}
C_{1}^{I} \exp \left(-j \alpha_{1} \omega s x_{3}\right) \\
C_{2}^{I} \exp \left(-j \alpha_{2} \omega s x_{3}\right) \\
C_{3}^{I} \exp \left(-j \alpha_{3} \omega s x_{3}\right) \\
C_{4}^{I} \exp \left(-j \alpha_{4} \omega s x_{3}\right)
\end{array}\right)
$$

where $C_{n}^{I}(n=1,2,3,4)$ is the weighted factor which determined by the boundary conditions.

Usually, the $\mathrm{SiO}_{2}$ and $\mathrm{FeNi}$ can be considered as isotropic media, and their typical matrix representation of acoustic wave equations are defined by

$$
\nabla_{i K} c_{K L}^{E} \nabla_{L j} u_{j}=\rho \frac{\partial^{2} u_{i}}{\partial t^{2}}, i, j=1,2,3, K, L=1,2, \ldots, 6,
$$

So, the integral solution in acoustic equation of the isotropic $\mathrm{SiO}_{2}$ film is assumed as

$$
u_{i}^{I I}\left(x_{1}, x_{3}\right)=A_{i}^{I I} \exp \left(-j \alpha_{p}^{I I} \omega s x_{3}\right)
$$

Then, substituting Equation (6) into the wave Equation (5), the corresponding Christoffel equation is obtained by

$$
\begin{gathered}
\left(\left(\alpha^{I I}\right)^{2} \boldsymbol{R}_{1}^{I I}+\alpha^{I I} \boldsymbol{R}_{2}^{I I}+\boldsymbol{R}_{3}^{I I}\right)\left(\begin{array}{c}
A_{1}^{I I} \\
A_{2}^{I I} \\
A_{3}^{I I}
\end{array}\right)=0 \\
\boldsymbol{R}_{1}^{I I}=\left(\begin{array}{ccc}
c_{44} & 0 & 0 \\
0 & c_{44} & 0 \\
0 & 0 & c_{11}
\end{array}\right), \boldsymbol{R}_{2}^{I I}=\left(\begin{array}{ccc}
0 & 0 & c_{44} \\
0 & 0 & 0 \\
c_{44} & 0 & 0
\end{array}\right), \boldsymbol{R}_{3}^{I I}=\left(\begin{array}{ccc}
c_{11}-\frac{\rho^{L}}{s^{2}} & 0 & 0 \\
0 & c_{44}-\frac{\rho^{L}}{s^{2}} & 0 \\
0 & 0 & c_{44}-\frac{\rho^{L}}{s^{2}}
\end{array}\right)
\end{gathered}
$$

Similarly, the conditions for existence of non-trivial solution in Christoffel equation is that the determinant of the coefficient matrix $(\Gamma)$ should be zero, that is, $\operatorname{det}(\Gamma)=0$. A six degree equation about $\alpha^{I}$ can be deduced. Since the thickness of the $\mathrm{SiO}_{2}$ film is limited, the six roots of $\alpha_{p}^{L}(p=1,2, \ldots, 6)$ all satisfy the Christoffel equation, and the integral form of the displacement in the frequency slowness domain is expressed by

$$
\widetilde{u}_{i}^{I I}\left(s, x_{3}\right)=\sum_{p=1}^{6} C_{p}^{I I}\left(A_{i}^{I I}\right)^{(p)} \exp \left(-j \alpha_{p}^{I I} \omega s x_{3}\right)
$$

where $i=1,2,3$. $A_{i}{ }^{I I}$ is eigenvectors corresponding to $a_{p}{ }^{I I}, C_{p}{ }^{I I}(p=1,2, \ldots, 6)$ are the weighted coefficients, and determined by the boundary conditions. 
The solution in FeNi thin film is similar to $\mathrm{SiO}_{2}$. And the corresponding integral solution of the displacement in the frequency slowness domain of the FeCo film can be expressed as

$$
\widetilde{u}_{i}^{I I I}\left(x_{1}, x_{3}\right)=\sum_{p=1}^{6} C_{p}^{I I I}\left(A_{i}^{I I I}\right)^{(p)} \exp \left(-j \alpha_{p}^{I I I} \omega s x_{3}\right)
$$

where, the corresponding six eigen values and eigenvectors in FeNi film are expressed as $a_{p}$ III and $A_{p} I I I$ $(p=1,2,3,4,5,6)$, and $C_{n}{ }^{I I I}(n=1,2,3,4)$ are the weighted coefficients.

There is no free charge distribution in the region of $0<x_{3}<h_{1}, h_{1}<x_{3}<h_{1}+h_{2}$, and $h_{1}+h_{2}<x_{3}$, which are marked by II, III and $G$, respectively. Then the potentials $\Phi^{I I}, \Phi^{I I I}$ and $\Phi^{G}$ satisfying the Laplace equation $\nabla^{2} \Phi\left(x_{1}, x_{3}\right)=0$ are introduced to meet the electrical boundary conditions, and the electric displacement components $D_{3}$ in the $x_{3}$ direction are determined in the corresponding regions. $\Phi^{I I}, \Phi^{I I I}, \Phi^{G}, D_{3}^{I I}, D_{3}^{I I I}$ and $D_{3}^{G}$ in the frequency slowness field are expressed by

$$
\left\{\begin{array}{l}
\widetilde{\Phi}^{I I}=B_{1}^{I I} \exp \left(-\omega s x_{3}\right)+B_{2}^{I I} \exp \left(\omega s x_{3}\right) \\
\widetilde{\Phi}^{I I I}=B_{1}^{I I I} \exp \left(-\omega s x_{3}\right)+B_{2}^{I I I} \exp \left(\omega s x_{3}\right) \\
\widetilde{\Phi}^{\mathrm{G}}=B^{G} \exp \left(-\omega s x_{3}\right) \\
\widetilde{D}_{3}^{I I}=-j k_{s}\left[j \varepsilon^{I I} B_{1}^{I I} \exp \left(-\omega s x_{3}\right)-j \varepsilon^{I I} B_{2}^{I I} \exp \left(\omega s x_{3}\right)\right] \\
\widetilde{D}_{3}^{I I}=-j k_{s}\left\{j \varepsilon^{I^{I I}} B_{1}^{I I I} \exp \left(-\omega s x_{3}\right)-j \varepsilon^{I I I} B_{2}^{I I I} \exp \left(\omega s x_{3}\right)\right\} \\
\widetilde{D}_{3}^{\mathrm{G}}=-j k_{s}\left[j \varepsilon_{0} B^{\mathrm{G}} \exp \left(-\omega s x_{3}\right)\right]
\end{array}\right.
$$

where, $\varepsilon^{I I}, \varepsilon^{I I I}$ and $\varepsilon_{0}$ are the dielectric constant of the $\mathrm{SiO}_{2}$ layer, $\mathrm{FeNi}$ layer and the air dielectric constant, respectively, $\varepsilon_{0}=8.854 \times 10^{-12}(\mathrm{~F} / \mathrm{m})$.

The boundary conditions for each surface of the layered media structure shown in Figure 2 are described as below. Firstly, the stress, displacement, and electric potential are continuous at $x_{3}=0$ and $x_{3}=h_{1}$, that is,

$$
\left\{\begin{array}{l}
\widetilde{T}_{3 j}-\widetilde{T}_{3 j}^{L}=0 \\
\widetilde{u}_{i}-\widetilde{u}_{i}^{L}=0 \\
\widetilde{\Phi}-\widetilde{\Phi}^{L}=0 \\
\widetilde{D}_{3}-\widetilde{D}_{3}^{L}=-\widetilde{\sigma}(s), i, j=1,2,3 \\
\widetilde{T}_{3 j}^{I I}-\widetilde{T}_{3 j}^{I I}=0 \\
\widetilde{u}_{i}^{I I}-\widetilde{u}_{i}^{I I}=0 \\
\widetilde{\Phi}^{I I}-\widetilde{\Phi}^{I I I}=0 \\
\widetilde{D}_{3}^{I I}-\widetilde{D}_{3}^{I I I}=0
\end{array}\right.
$$

The normal component of the stress at $x_{3}=h_{1}+h_{2}$ is considered as zero, and the corresponding displacement and electric potential are continuous as

$$
\left\{\begin{array}{c}
\widetilde{T}_{3 j}^{I I I}=0 \\
\widetilde{\Phi}^{I I I}-\widetilde{\Phi}^{\mathrm{G}}=0 \\
\widetilde{D}_{3}^{I I I}-\widetilde{D}_{3}^{\mathrm{G}}=0
\end{array}\right.
$$

To sum up, there are 21 undetermined unknowns in the Christoffel equation expressed by $C_{n}^{I}(n=1,2,3,4), C_{p}^{I I}(p=1,2,3,4,5,6), C_{p}^{I I I}(p=1,2,3,4,5,6), B_{1}^{I I}, B_{2}^{I I}, B^{\mathrm{G}}, B_{1}^{I I I}$ and $B_{2}^{I I I}$. It means that there are 21 equations to determine the unknowns. To simply the calculation, the approach of effective dielectric constant was used.

The effective dielectric constant at the boundary of $x_{3}=0$ is defined by [22]

$$
\varepsilon_{s}(s)=\frac{\widetilde{\sigma}(s)}{\left.2 \pi f|s| \widetilde{\Phi}\left(s, x_{3}\right)\right|_{x_{3}=0}}
$$


Accordingly, the $\varepsilon_{s}(s)$ value corresponding different slowness $s$ can be deduced, and also the surface effective dielectric constant $\varepsilon_{s}(s)$ curve can be pictured. Usually, $\varepsilon_{s}(s)$ is a complex number. The slowness $s_{m}$ corresponding to the maximum value of the real part shows the case of metallization at $x_{3}=0$. While the slowness $s_{f}$ corresponding to the zero-crossing of the real part shows the free surface at $x_{3}=0$. According to the reciprocal relationship, the corresponding SAW velocity in metalized surface $v_{m}$, and velocity in free surface, $v_{f}$, at $x_{3}=0$, can be obtained, respectively.

Usually, the magnetostriction of FeNi film will be changed by its aspect ratio [23],

$$
\beta=\beta_{0}\left(1+p \cdot k_{p}\right)
$$

where $\beta$ and $\beta_{0}$ are the perturbed and unperturbed magnetostriction of the FeNi film, respectively, and $p=l / w$ is the aspect ratio, $l$ and $w$ are the length and width of FeNi film, respectively. $k_{p}$ is the correlation coefficient, and is set to $7.13 \times 10^{-4}$ [23].

Owing to the magnetostrictive nature, the FeNi film would be magnetized under the electromagnetic field, resulting in the changes in FeNi film thickness $h$ and density $\rho$, which can be written by

$$
\left\{\begin{array}{l}
h=h_{2}\left(1-\frac{\beta}{2}\right) \\
\rho=m /\left[a_{0} \times\left(1+\frac{\beta}{2}\right) \times b_{0} \times\left(1-\frac{\beta}{2}\right) \times h_{2} \times\left(1-\frac{\beta}{2}\right)\right]
\end{array}\right.
$$

where $m$ is the mass of the FeNi film, $a_{0}, b_{0}, h_{2}$ are the initial length, width and thickness of unperturbed $\mathrm{FeNi}$, respectively, and obviously $m=\rho^{I I I} \times\left(a_{0} \times b_{0} \times h_{2}\right)$.

Moreover, the young's modulus $(E)$ of the FeNi will also be modulated by the electromagnetic field intensity $(H)$, and can be taken experimentally by using the laser resonance method [24]. Correspondingly, the elastic coefficient of the FeNi will also be expressed as

$$
\left\{\begin{array}{c}
c_{11} F=\frac{E \times\left(1-u_{F}\right)}{\left(1+u_{F}\right)\left(1-2 u_{F}\right)} \\
c_{12} F=\frac{E \times u_{F}}{\left(1+u_{F}\right)\left(1-2 u_{F}\right)} \\
c_{44}{ }^{F}=\frac{E \times\left(1-2 u_{F}\right)}{2\left(1+u_{F}\right)\left(1-2 u_{F}\right)}
\end{array}\right.
$$

where the Poissoncoefficient of $\mathrm{FeNi}, u_{F}$, was considered as constants irrelevant to the electromagnetic field, and it was set to 0.3 .

Hence, using above equations and the measured relationship between the young's modulus of FeNi and the magnetic field (Figure 3), the shift in SAW velocity shift depending on the applied electromagnetic field can be calculated. Also, considering the relationship between the electromagnetic field intensity $(H)$ and current (A) described by the Biot-Savart law [25],

$$
H=\mu_{0} I /(4 \pi l)
$$

where, $\mu_{0}$ denotes the permeability in vacuum. $l$ expresses the distance between the sensor chip and wires, and is set to $1 \mathrm{~cm}$ in this calculation, the sensitivity in current sensing can be evaluated theoretically. Furthermore, the influence of FeNi thickness and aspect ratio $\left(a_{0} / b_{0}\right)$ on sensor response can be analyzed using the physical parameters listed in Table 1. The sensor response was defined as the difference between the perturbed and unperturbed SAW velocity. The given electromagnetic field intensity and working frequency of sensor in the calculation are set to 200 Oe and $150 \mathrm{MHz}$. Nonlinear relationship was observed between the sensor response and FeNi thickness, as shown in Figure 4a. The sensor response increases rapidly with increasing the FeNi thickness up to $\sim 0.5 \mu \mathrm{m}$ and then reaches the max response value. Hence, the optimal value in FeNi thickness is advised to $\sim 500 \mathrm{~nm}$. Additionally, the effect of the aspect ratio of the FeNi on sensor response was also analyzed as shown in Figure $4 \mathrm{~b}$. With increasing the aspect ratio (1:1 to 2:1), larger sensor response was observed. However, lager aspect ratio will lengthen SAW propagation path, resulting in increase of the acoustic 
propagation attenuation. So, in our opinion, proper increase in aspect ratio of FeNi film is an effective way to improve the sensor sensitivity.

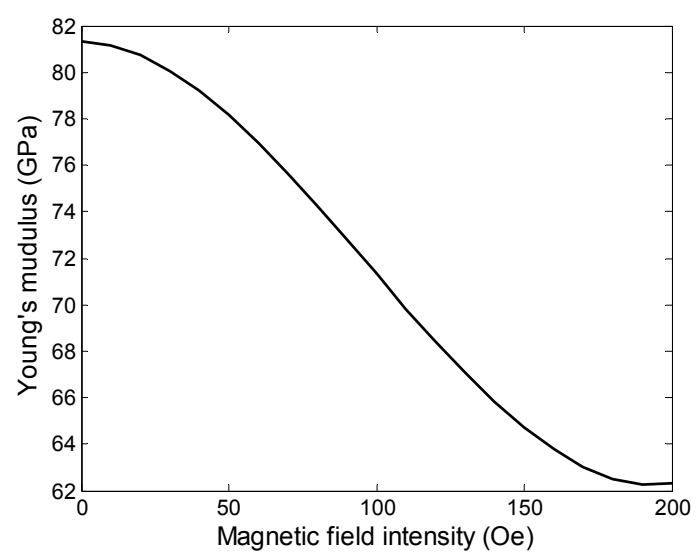

Figure 3. Measured relationship between the young modulus of FeNi and magnetic field intensity.

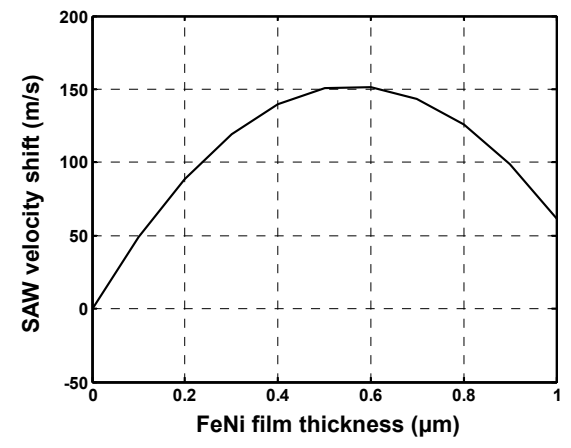

(a)

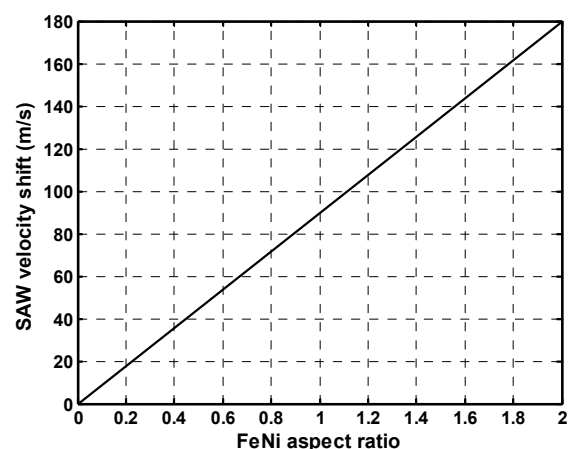

(b)

Figure 4. The calculated relationship among the sensor response and the FeNi film thickness (a), and the aspect ratio (b), where the electromagnetic field intensity is set to 200 Oe.

Then, referring to the Biot-Savart law mentioned Equation (16) and velocity-frequency relationship of $\Delta \mathrm{v} / \mathrm{v}_{\mathrm{s}}=\Delta \mathrm{f} / \mathrm{f}_{0}\left(\Delta \mathrm{v}\right.$ and $\Delta \mathrm{f}$ are the shift in SAW velocity $\mathrm{v}_{\mathrm{s}}$ and sensor frequency $\left.\mathrm{f}_{0}\right)$, the current sensitivity of the $150 \mathrm{MHz}$ SAW sensor coated with $500 \mathrm{~nm}$ FeNi (aspect ratio is set to 2:1) was calculated as shown in Figure 5, and sensitivity of $\sim 11 \mathrm{KHz} / \mathrm{A}$ was expected.

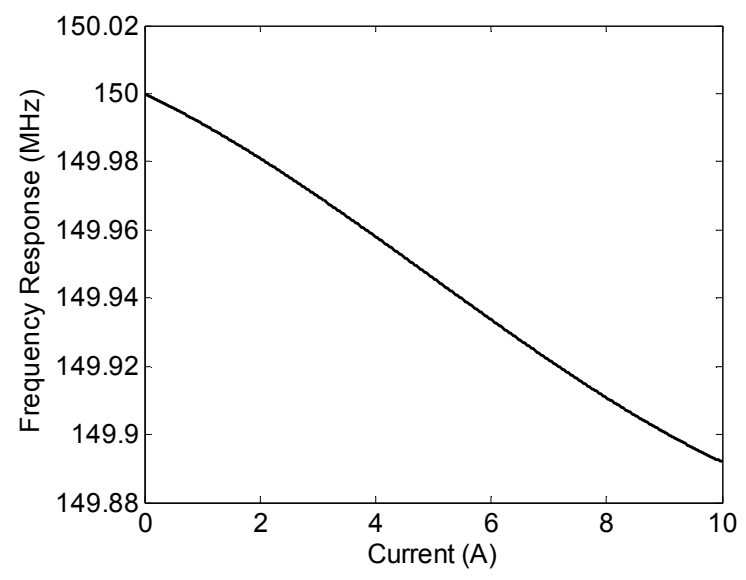

Figure 5. The calculated sensor response of FeNi coated SAW current sensor. 


\section{Technique Realization}

\subsection{Sensor Chip}

Two SAW delay lines with two photolithographically defined $100 \mathrm{~nm}$ Al interdigital transducers (IDTs) separated by a $2.5 \mathrm{~mm}$ length of propagation path were reproducibly fabricated on a same piezoelectric $\mathrm{YZ} \mathrm{LiNbO}_{3}$ wafer. The launch and receive SPUDT pairs were set to 60 and 30, respectively. The acoustic aperture is $100 \lambda$ ( $\lambda$ stands for the wavelength corresponding operation frequency of $150 \mathrm{MHz}: 23.19 \mu \mathrm{m})$. Following the preparation of delay line patterns, $\mathrm{a} \mathrm{SiO}_{2}$ thin film $(50 \mathrm{~nm})$ was deposited on the SAW device surface to protect the electrodes in process of FeNi coating by using the plasma enhanced chemical vapor deposition (PECVD). Prior to FeNi coating, the $\mathrm{SiO}_{2}$ film surface was cleaned of any contaminants by a routine cleaning procedure involving rinsing in acetone solution, a DI water rinse and drying by $\mathrm{N}_{2}$. Then, the magetostrictive FeNi film with various aspect ratio (1:1, 1.5:1 and 2:1) was deposited onto the sensing device surface by RF magnetron sputtering (JGP-560C magnetron sputtering equipment) and overlay technique from a FeNi target (nominal composition). The base pressure is $2 \times 10^{-4} \mathrm{~Pa}$. The Ar was used as the sputtering gas, and corresponding pressure is set to $1 \mathrm{~Pa}$. The power density of the target was $400 \mathrm{~V}(\mathrm{DC}) \times 80 \mathrm{~mA} / \mathrm{p}(35 \mathrm{~mm})^{2}$. Various FeNi thicknesses (300, 500 and $700 \mathrm{~nm}$ ) were obtained by controlling the sputtering time. The specific technological process is depicted in Figure 6. Figure 7 is the picture of the developed FeNi-coated sensor chip, and FeNi coating was indicated by the dark area. The surface morphology of FeNi coating was observed by JSM5600-Lv scanning electron microscopy (SEM) (JEOL, Tokyo, Japan), as shown in Figure 8. It can be seen that the prepared FeNi film are densely and uniformly distributed. To make the comparison, $500 \mathrm{~nm}$ FeCo film with aspect ratio of 2:1 was also deposited onto the similar SAW device surface to form a FeCo-coated sensor.

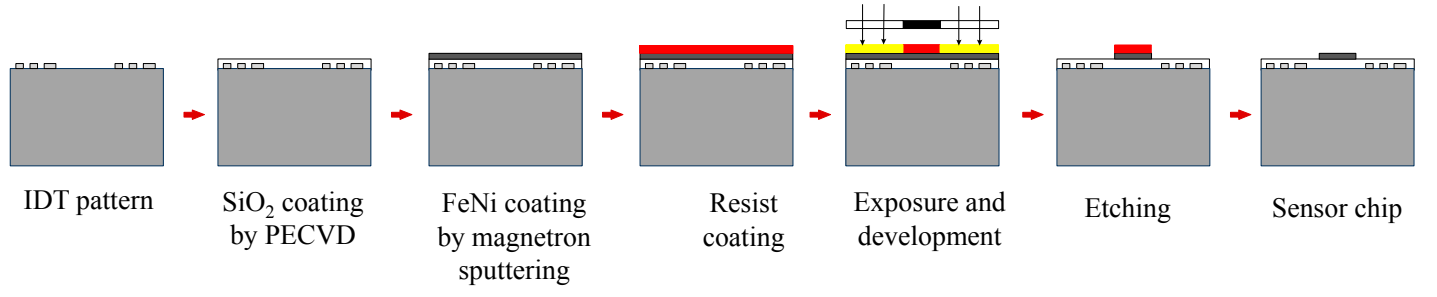

Figure 6. The technological process of SAW current sensor chip. IDT: interdigital transducer; PECVD: plasma enhanced chemical vapor deposition.

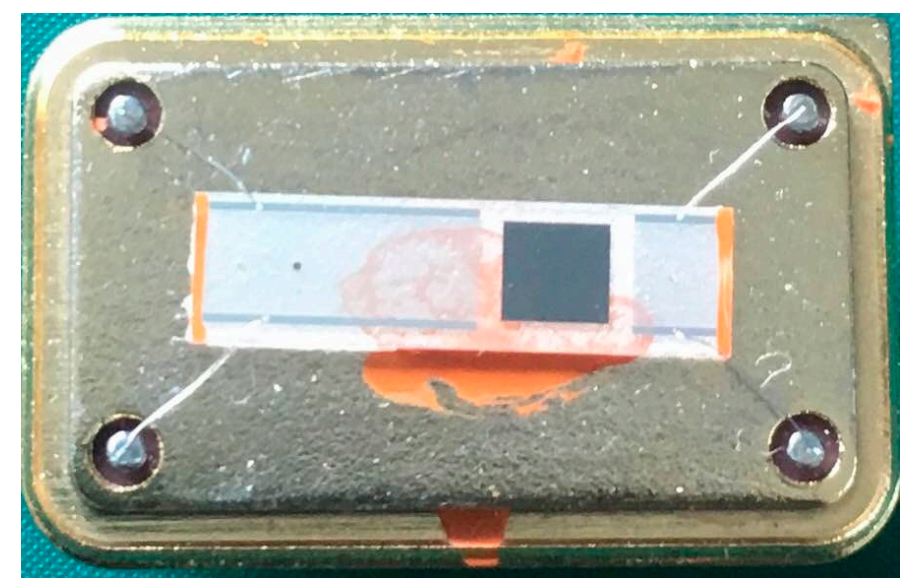

Figure 7. The physical picture of developed sensor chip, FeNi thickness: $500 \mathrm{~nm}$, aspect ratio: 1:1. 


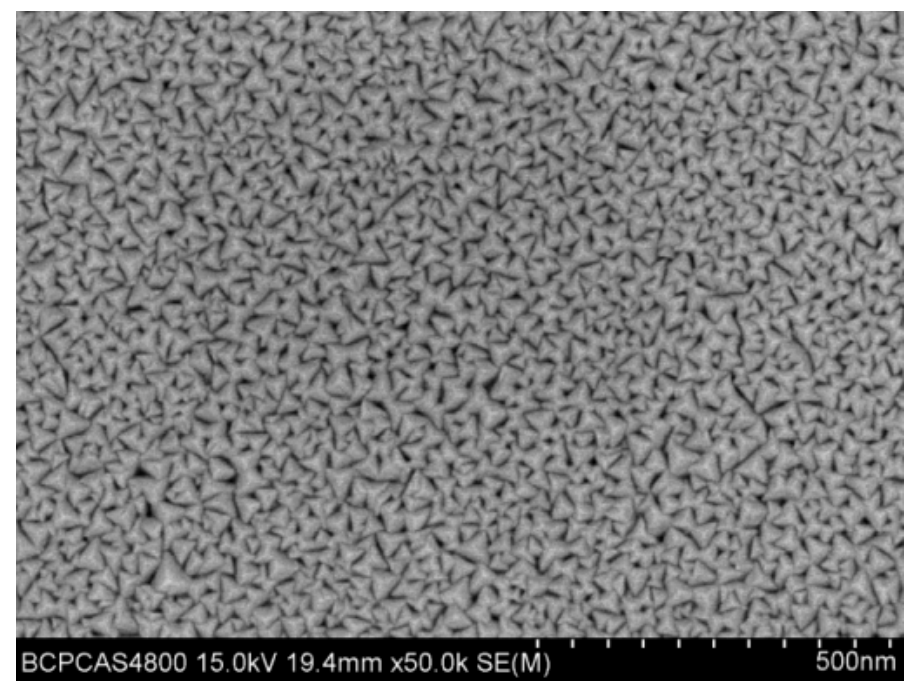

Figure 8. The observed surface morphology of the FeNi coating.

Next, the FeNi-coated SAW sensor chips with various FeNi thickness (300, 500 and $700 \mathrm{~nm}$ ) were characterized by using the network analyzer, as shown in Figure 9. With increases in FeNi thickness, the operation frequency of the sensor chip decreases owing to the mass loading effect from the FeNi coating. The measured operation frequency and insertion loss of the sensor chip with 300, 500 and $700 \mathrm{~nm}$ FeNi (with aspect ratio of 2:1) are $149.9 \mathrm{MHz}$ and $5 \mathrm{~dB}, 149.7 \mathrm{MHz}$ and $7 \mathrm{~dB}, 149.6 \mathrm{MHz}$ and $8 \mathrm{~dB}$, respectively.

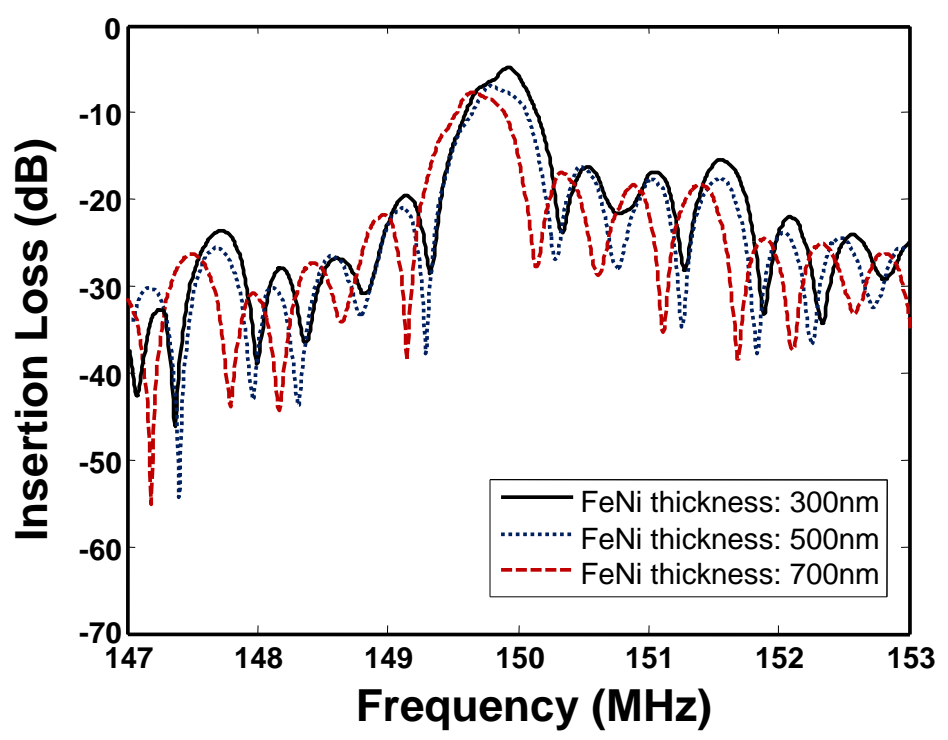

Figure 9. Measured frequency response of the FeNi coated sensor chip.

\subsection{FeNi Coated Current Sensor}

After the preparation of the FeNi coated sensor chip, the launch and receive transducers of the SAW chips were connected to the oscillation loop composed of amplifier, phase shifter, and mixer, and forms the differential oscillator, as shown in Figure 1. To improve the frequency stability of the oscillator, the oscillation was tuned on the frequency corresponding to lowest insertion loss of the SAW device by adjusting the inductor or capacitor values in phase shifter. The produced differential frequency signal was connected at 20 points per second by the frequency acquisition module (FAM) 
made by field programmable gate arrat (FPGA), gathered and plotted by the personal computer in real time. Due to the magetostrictive effect, the FeNi produces the magnetostrictive strain and $\Delta \mathrm{E}$ effect, where the thickness, density and young modulus of the FeNi film are changed accordingly, and then the corresponding changes in differential oscillation frequency signal was picked to evaluate the current to be measured.

\section{Sensor Experiment and Discussion}

\subsection{Experimental Setup}

The physical map and schematic diagraph of the experimental setup for current sensor characterization were shown in Figure 10. The experimental setup was composed of the developed SAW current sensor, DC current source (YangZhou Huatai Electronics Co., Ltd., Yangzhou, China), Helmholtz coil (Tianduan Industry Co., Ltd, Shanghai, China) for stimulating the magnetic field under giving current, PC and Gauss meter (Tianduan Industry Co., Ltd) for calibration (Figure 10a). Usually, the strongest magnetic field intensity and the best uniformity occur at the central axis of the Helmholtz coil, hence, the sensor was positioned at the central axis of the platform in the middle of the coil. As shown in Figure 10b, the applied current for Helmholtz coil stimulates electromagnetic field, and the corresponding sensor response induced by the magnetostrictive effect was taken from the oscillation frequency signal connected at 1 point per $50 \mathrm{~ms}$ by the FPGA.

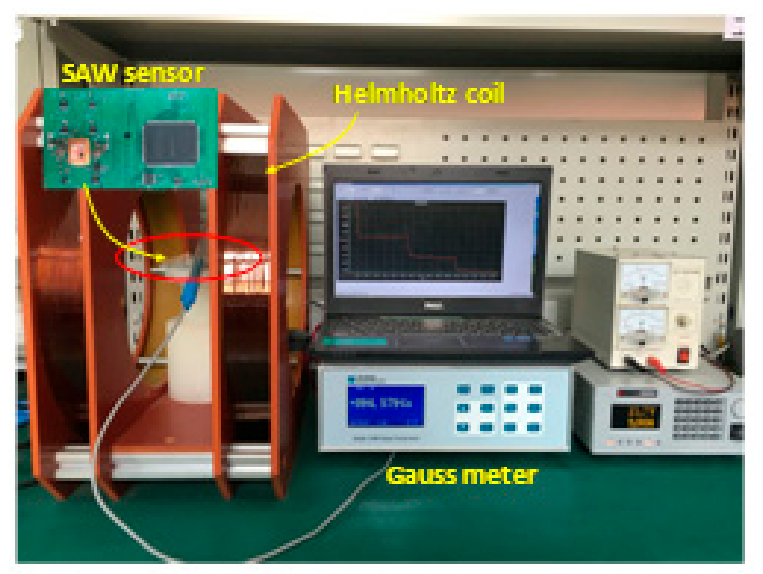

(a)

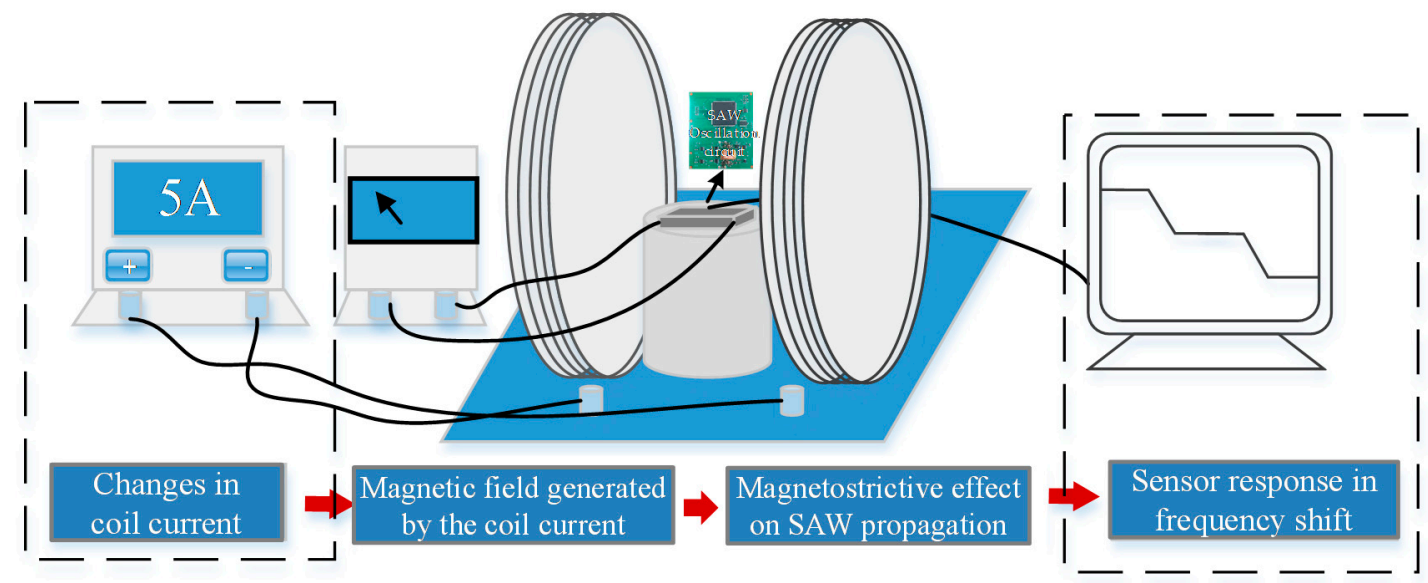

(b)

Figure 10. The physical picture and schematic diagraph of the experimental setup for sensor performance evaluation, (a) experimental setup; (b) working principle. 


\subsection{Baseline Noise Measurement}

Prior to sensor performance evaluation, the baseline noise of the develop sensor was tested by measuring the frequency stability of the differential oscillator, as shown in Figure 11. Excellent short-term frequency stability of $\pm 0.5 \mathrm{~Hz} / \mathrm{s}$ and medium-term frequency stability of $\pm 5 \mathrm{~Hz} / \mathrm{h}$ were obtained.

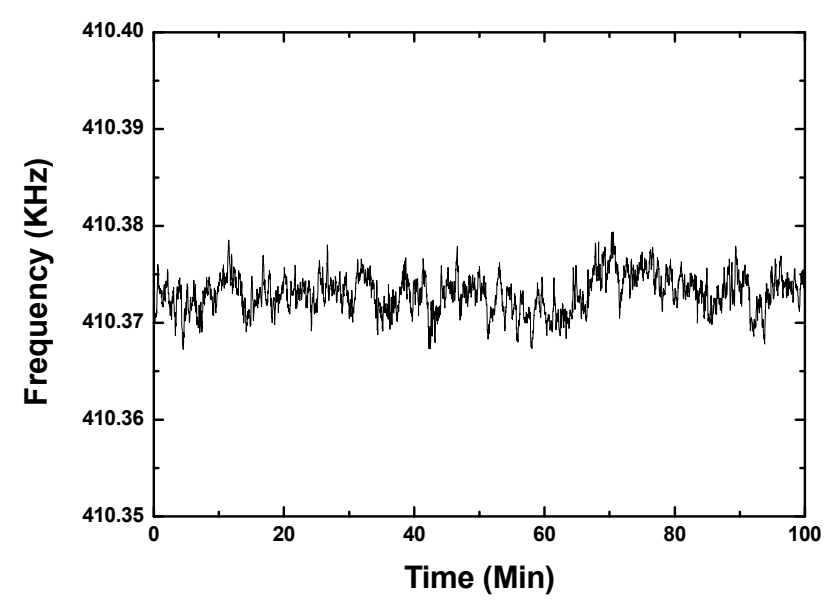

Figure 11. The baseline noise measurement of the developed sensor.

\subsection{Hysteresis Measurement}

The hysteresis loops of the FeNi and FeCo were characterized by using the resistance strain gauge method [20], as depicted in Figure 12. The horizontal and vertical axes in the graph stand for external magnetic field intensity and magnetostrictive coefficient respectively. The curves 1 and 2 represent the corresponding changes in the magnetostriction of the FeNi coating at increasing and decreasing magnetic field intensity, respectively. Meanwhile, the curves 3 and 4 characterize the relationship between the magnetostrictive coefficient of the $\mathrm{FeCo}$ and the external magnetic field. It is clear that the hysteresis loop of FeNi and FeCo were distinctly different. Owing to the week remanence and hysteresis effect, the FeNi alloy exhibits relatively narrow and steep hysteresis loop over the FeCo, which means the FeNi coated sensor will offer better symmetry in current sensing. That is the reason why the FeNi was favored as the magneticsensitive interface for magnetic sensing.

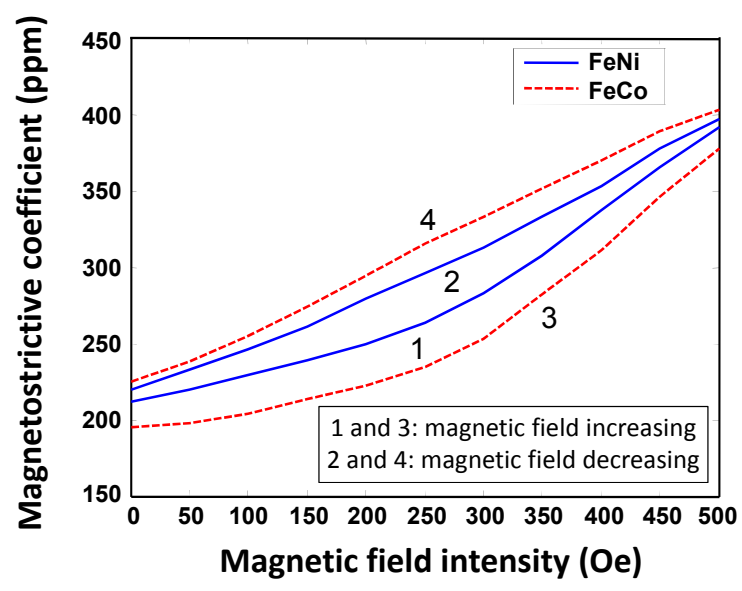

Figure 12. The measured magnetostrictive curves for FeCo and FeNi. 


\subsection{FeNi Film Thickness and Aspect Ratio Effect on Sensor Performance}

The sensor response of the developed $150 \mathrm{MHz}$ SAW sensors with different FeNi film aspect ratio $(1: 1,1.5: 1$, and 2:1) and various FeNi film thickness $(300,500$, and $700 \mathrm{~nm})$ were characterized at current range of $0 \sim 10 \mathrm{~A}$ and room temperature $\left(20^{\circ} \mathrm{C}\right)$, as concluded in Figure 13. Figure 13a indicates the sensor sensitivity for different FeNi film aspect ratio (1:1, 1.5:1, and 2:1) was measured to be 8.4, 9.7, and $10.7 \mathrm{KHz} / \mathrm{A}$. Obviously, larger aspect ratio of FeNi film is effective to improve the sensor sensitivity. On the other hand, the effect on sensor sensitivity from the FeNi film thickness is relatively complicated, as shown in Figure 13b. Compared to the sensors with FeNi film thickness of 300 and $700 \mathrm{~nm}$, the senor with a $500 \mathrm{~nm}$ thickness FeNi film has highest sensitivity, which agrees well with the calculated result mentioned in Figure 4a. Therefore, the optimal FeNi thickness and aspect ratio were determined to be $\sim 500 \mathrm{~nm}$ and 2:1, respectively.

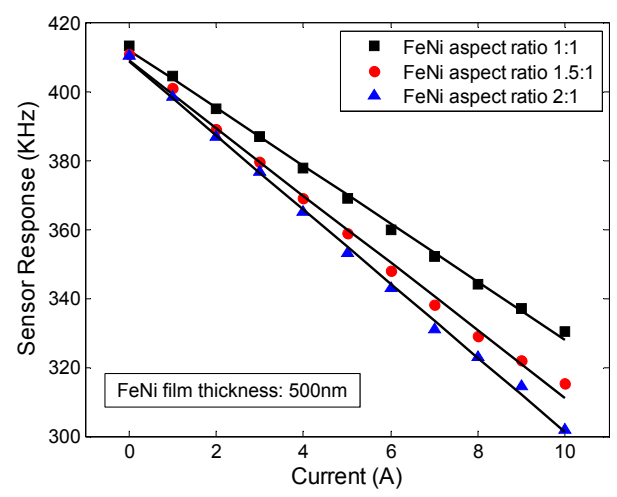

(a)

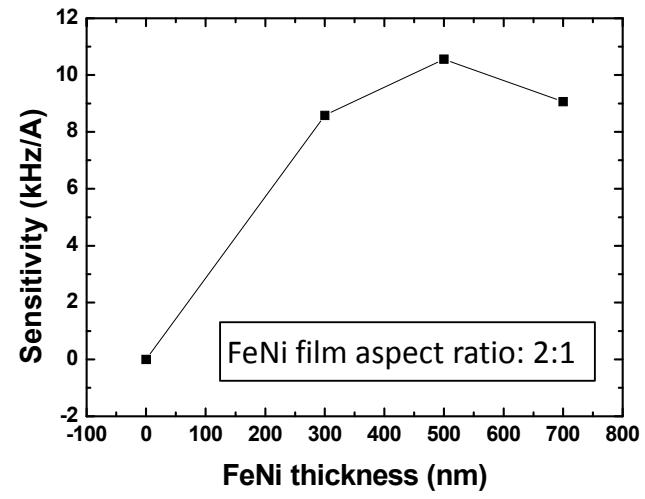

(b)

Figure 13. The effect on sensor response from the FeNi aspect ratio (a) and FeNi thickness (b).

\subsection{Repeatability Test}

Response and recovery times for current sensing were tested by using the developed FeNi coated current sensor with FeNi aspect ratio of 2:1 and thickness of $500 \mathrm{~nm}$. Sensor response measurements were performed for three consecutive 40-s exposures to current at intensity from $9 \mathrm{~A}$ to $10 \mathrm{~A}$, as shown in Figure 14. When the current was varied from $9 \mathrm{~A}$ to $10 \mathrm{~A}$, the frequency response showed a rapid rise (only one sampling point) to its steady-state value. When the current was decreased to $9 \mathrm{~A}$, the frequency response rapidly fell to its initial value. From this promising result, we hypothesize that the developed sensor exhibits excellent repeatability and fast response.

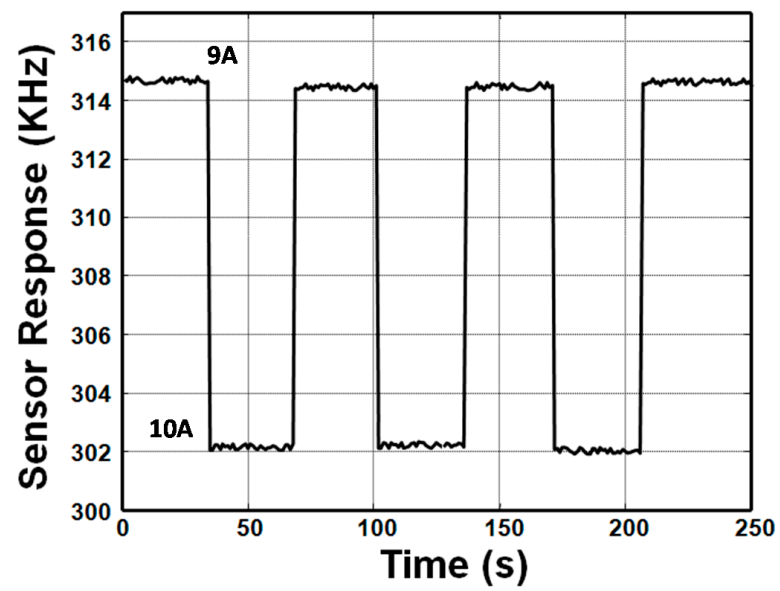

Figure 14. The repeatability test of the developed SAW sensor. 


\subsection{Symmetry and Sensitivity Evaluation}

Then, the symmetry of the developed current sensor was the main concern in this work, and measured as shown in Figure 15. It is obviously that the gathered sensor signal decreases monotonically with increasing current intensity from 0 to $10 \mathrm{~A}$, meanwhile, it increases with decreasing current intensity, and reaches to the initial value. It means that good symmetry in sensor response was observed for the FeNi coated current sensor.

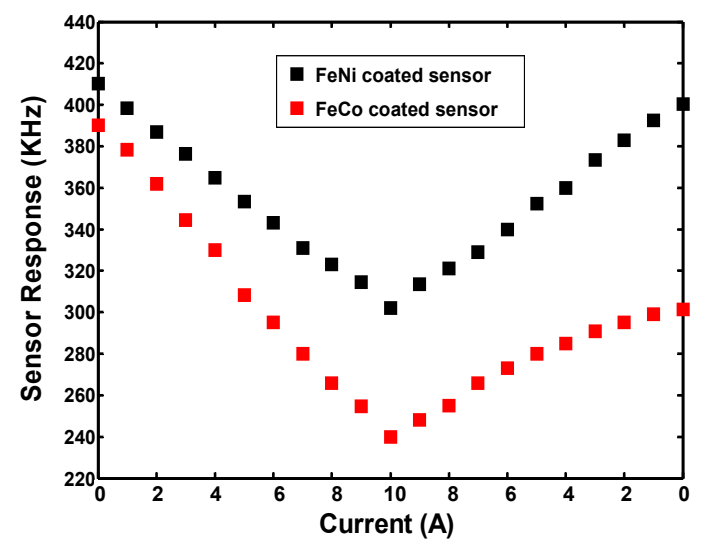

Figure 15. The symmetry test of the FeNi coated sensor and in comparison to FeCo coated sensor.

Also, for comparison, the symmetry of the developed FeCo coated current sensor [12] was measured as shown in Figure 15. The obvious asymmetry phenomenon was observed in the sensor response at increasing and decreasing current intensity, which is caused by its larger remanence and hysteresis effect over the FeNi described in Figure 3.

Usually, the hysteresis error $\left(\varepsilon_{\mathrm{H}}\right)$ was defined by $\varepsilon_{H}=\frac{(\Delta y)_{\max }}{2 y_{F S}} \times 100 \%,(\Delta y)_{\max }=\max \left(y_{u i}-y_{d i}\right)$, where $y_{u i}$ and $y_{d i}$ are the response at the same current input when the current increases and decreases, $y_{F S}$ is the full-scale output of the current sensor. Hence, a hysteresis error of $0.97 \%$ was obtained from the FeNi coated sensor, which is substantially lower than the FeCo coated sensor (8.72\%).

The corresponding sensitivity was measured to be $10.7 \mathrm{KHz} / \mathrm{A}$, which agrees well with the predicted value of $11 \mathrm{KHz} / \mathrm{A}$, as shown in Figure 16, and it corresponds to a magnetic field sensitivity of $5.35 \mathrm{KHz} / \mathrm{Oe}$. Usually, the detection limit in current sensing closely relates to the frequency stability of the oscillator. Considering the measured short-term stability of $\pm 0.5 \mathrm{~Hz} / \mathrm{s}$, and $10.8 \mathrm{KHz}$ in response to current of $1 \mathrm{~A}$, the threshold detection limit of the developed sensor was expected to be $\sim 0.2 \mathrm{~mA}$. From these results, we suggest that this prototype FeNi coated SAW sensor is very promising for current sensing with high sensitivity, excellent repeatability, good symmetry and linearity.

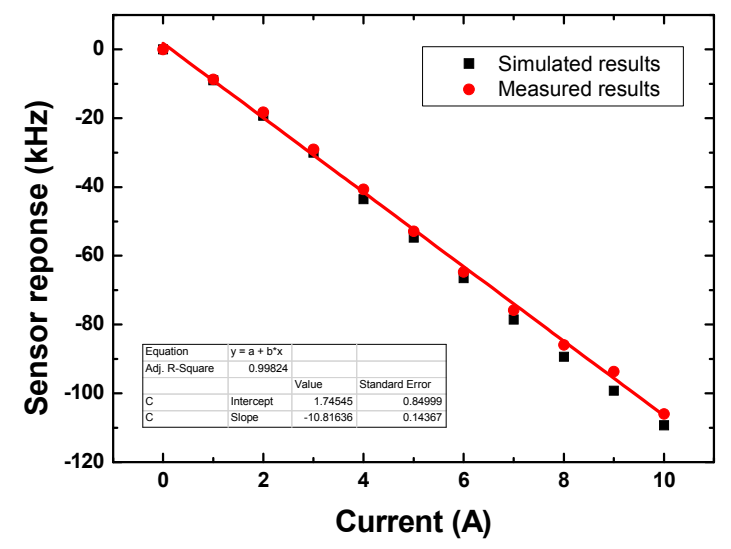

Figure 16. The sensitivity evaluation of the developed FeNi coated sensor. 


\section{Conclusions}

To improve the symmetry in sensor response, a magnetostrictive FeNi film was used to construct the SAW based current sensor. The optimal design parameters were determined theoretically prior to sensor development. High sensitivity of $10.7 \mathrm{KHz} / \mathrm{A}$, low detection limit of $0.2 \mathrm{~mA}$, excellent linearity of $1.22 \%$, and lower hysteresis error of $0.97 \%$ in sensor response were achieved from the developed $150 \mathrm{MHz}$ FeNi coated SAW current sensor, in which, the FeNi film thickness and aspect ratio was designed to $500 \mathrm{~nm}$ and 2:1, respectively.

Acknowledgments: This work was supported by National Natural Science Foundation of China (No. 11374254) and technological project of State Grid Corporation of China (DG71-15-038/5442DG150031).

Author Contributions: Jie Tong and Wen Wang conceived and designed the experiments; Yana Jia designed and fabricated the sensor chips; Yang Wang performed the experiments; Shiyue Wang conducted the experimental data analysis; Xinlu Liu performed the data collection; Yuqing Lei drew the figures; Wen Wang wrote the paper.

Conflicts of Interest: The authors declare no conflict of interest.

\section{References}

1. Tang, Y.L.; Li, Z.J.; Ma, J.Y. Ammonia gas sensors based on $\mathrm{ZnO} / \mathrm{SiO}_{2}$ bi-layer nanofilms on ST-cut quartz surface acoustic wave devices. Sens. Actuators B 2014, 201, 114-121. [CrossRef]

2. Ji, X.; Fan, Y.; Chen, J.; Han, T.; Cai, P. Passive wireless torque sensor based on surface transverse wave. IEEE Sens. J. 2016, 16, 888-894. [CrossRef]

3. Wang, W.; Huang, Y.; Liu, X.L.; Liang, Y. Surface acoustic wave acceleration sensor with high sensitivity incorporating ST-X quartz cantilever beam. Smart Mater. Struct. 2015, 24, 015015. [CrossRef]

4. Ma, G.M.; Wu, Z.; Zhou, H.Y.; Jiang, J. A wireless and passive on-line temperature monitoring system for GIS based on surface acoustic wave sensor. IEEE Trans. Power Deliv. 2016, 31, 1270-1280. [CrossRef]

5. Stoney, R.; Geraghty, D.; Odonnell, G.E. Characterization of differentially measured strain using passive wireless surface acoustic wave strain sensors. IEEE Sens. J. 2014, 14, 722-728. [CrossRef]

6. Kadota, M.; Shigeo, I.; Yoshihiro, I.; Takuo, H.; Kenjiro, O. Magnetic sensor based on surface acoustic wave resonators. Jpn. J. Appl. Phys. 2011, 50, 07HD07. [CrossRef]

7. Kadota, M.; Shigeo, I. Sensitivity of surface acoustic wave magnetic sensors composed of various Ni electrode structures. Jpn. J. Appl. Phys. 2012, 51, 07GC21. [CrossRef]

8. Meriem, E.; Omar, E.; Abdelkrim, T.; Keltouma, A.A.; Laurent, B.; Frederic, S. FEM modeling of multilayer piezo-magnetic structure based surface acoustic wave devices for magnetic sensor. Procedia Eng. 2014, 87, $408-411$.

9. Meriem, E.; Omar, E.; Sebastien, P.W.; Laurent, B.; Sergei, Z. Magnetic field SAW sensors based on magnetostrictive-piezoelectric layered structures: FEM modeling and experimental validation. Sens. Actuators A Phys. 2016, 240, 41-49.

10. Li, W.; Dhagat, P.; Jander, A. Surface acoustic wave magnetic sensor using galfenol thin film. IEEE Trans. Magn. 2012, 48, 4100-4102. [CrossRef]

11. Zhou, H.; Talbi, A.; Tiercelin, N.; Matar, O.B. Multilayer magnetostrictive structure based surface acoustic wave devices. Appl. Phys. Lett. 2014, 104, 114101. [CrossRef]

12. Wang, W.; Jia, Y.; Liu, X. Enhanced sensitivity of temperature-compensated SAW based current sensor using the magnetostrictive effect. Smart Mater. Struct. 2017, 26, 025008. [CrossRef]

13. Hauser, H.; Steindl, R.; Hausleitner, C.; Pohl, A.; Nicolics, J. Wirelessly interrogable magnetic field sensor utlizing giant magneto-impedance effect and surface acoustic wave devices. IEEE Trans. Instrum. Meas. 2000, 49, 648-652. [CrossRef]

14. Li, B.; Morsy, A.M.; Kosel, J. Ooptimization of autonomous magnetic field sensor consisting of giant magnetoimpedance sensor and surface acoustic wave transducer. IEEE Trans. Magn. 2012, 48, 4324-4327. [CrossRef]

15. Li, B.; Yassine, O.; Kosel, J. A surface acoustic wave passive and wireless sensor for magnetic fields, temperature, and humidity. IEEE Sens. J. 2015, 15, 453-462. [CrossRef] 
16. Steindl, R.; Hausleimer, C.; Hauser, H. Wireless magnetic field sensor employing SAW-transponder. In Proceedings of the 2000 12th IEEE International Symposium on Applications of Ferroelectrics 2000 (ISAF 2000), Honolulu, HI, USA, 21 July-2 August 2000; pp. 855-858.

17. Reindl, L. Wireless Passive SAW Identification Marks and Sensors. In Proceedings of the 2nd International Symposium Acoustic Wave Devices for Future Mobile Communication Systems, Chiba, Japan, 3-5 March 2004; pp. 1-12.

18. Wang, W.; He, S.; Li, S. Enhanced Sensitivity of SAW Gas Sensor Coated Molecularly Imprinted Polymer Incorporating High Frequency Stability Oscillator. Sens. Actuators B Chem. 2007, 125, 422-427.

19. Plessky, V.P.; Koskela, J. Coupling-of-modes analysis of SAW devices. Int. J. High Speed Electr. Syst. 2000, 10, 1-81. [CrossRef]

20. Auckenthaler, R.; Carey, M.; Lioy-Thomas, H. Score normalization for text-independent speaker verification systems. Digit. Signal Process. 2000, 10, 42-54. [CrossRef]

21. Auld, B.A. Acoustic Fields and Waves in Solids; Wiley: New York, NY, USA, 1973; Volume 1.

22. Milsom, R.F.; Reilly, N.; Redwood, M. Analysis ofgeneration and detection of surface and bulk acoustic waves by interdigital transducers. IEEE Trans. Sonics Ultrason. 1977, 24, 147-166. [CrossRef]

23. Cao, D.; Jin, C.; Pan, L. Magnetic Properties and Microstructure Investigation of FeNi Films with Step-Height by Nano-MOKE. IEEE Trans. Magn. 2015, 51, 2005004. [CrossRef]

24. Morales, A.L.; Nieto, A.J. Simultaneous Measurement of Young's Modulus and Damping Dependence on Magnetic Fields by Laser Interferometry. In Proceedings of the International Conference on Thermal, Mechanical and Multi-Physics Simulation Experiments in Microelectronics and Micro-Systems, London, UK, 16-18 April 2007; pp. 1-5.

25. Pyati, V.P. Simplified Biot-Savart Law for Planar Circuits. IEEE Trans. Educ. 1986, E-29, 32-33. [CrossRef]

(C) 2017 by the authors. Licensee MDPI, Basel, Switzerland. This article is an open access article distributed under the terms and conditions of the Creative Commons Attribution (CC BY) license (http:/ / creativecommons.org/licenses/by/4.0/). 